\title{
Investigation of ketoacidurias by two-dimensional paper chromatography
}

\author{
R. F. COWARD, P. SMITH, AND J. W. T. SEAKINS \\ From the RAF Institute of Aviation Medicine, Farnborough, Hants, \\ and the Institute of Child Health, London
}

SYNOPSIS A simple paper chromatographic method was found to be effective for the study of phenylketonuria and tyrosyluria. It proved to be much more reliable than conventional tests for the detection of abnormal amounts of ketoacids in urine and was suitable and convenient for following the effects of dietary variations on the excretion of these compounds. Information obtained about ketones other than those of primary importance to the conditions studied included confirmation of the excretion of $p$-hydroxyphenylpyruvic acid in phenylketonuria. A modification of the method applicable to histidinaemia was devised.

In several conditions, including phenylketonuria, various types of tyrosyluria, histidinaemia, and maple syrup urine disease, excretion of a-ketoacids is of primary importance. Detection of these acids may be virtually essential in connexion with diagnosis and a convenient method of monitoring their excretion may be desirable in studies involving variation of dietary conditions, especially where this constitutes an integral part of treatment.

Investigations of the excretion of ketoacids themselves have hitherto been somewhat limited owing to the lack of suitable simple techniques; more usually, related compounds such as aminoacids or hydroxyacids have been the subject of studies. A fundamental difficulty hampering the direct investigation of the ketoacids is the general instability and reactivity of these substances. However, they may be readily stabilized by reaction with 2,4-dinitrophenylhydrazine (DNPH) reagent to give sparingly watersoluble, yellow 2,4-dinitrophenylhydrazones (DNPs), ketoacidurias have often been detected in the first place as a result of the formation of a visible precipitate on adding DNPH reagent to urine. Paper chromatography of ketoacid DNPs has attracted considerable attention. Notable advantages include the ease with which the coloured spots may be dztected and, if desired, eluted from paper and measured quantitatively by spectrophotometric methods. However, no completely satisfactory general method applicable to urine analysis has

Received for publication 16 October 1968 resulted when orthodox paper chromatographic methods have been employed, largely because the tendency of many DNPs to yield multiple spots has prevented the development of two-dimensional techniques.

It has recently been shown (Smith, 1967) that ketoacid DNPs chromatograph well on Whatman no. SG81 (silica gel-impregnated) paper and a twodimensional method applicable to biological fluids, including urine, has been reported (Coward and Smith, 1968). Applications of this new technique to cases of phenylketonuria and tyrosyluria are now reported and modifications to the procedure necessary in the case of histidinaemia are described.

\section{METHODS}

If possible urines were examined immediately after voiding; otherwise 24-hour samples were collected over acid and processed immediately upon receipt. The urine $(10 \mathrm{ml})$ was treated with an equal volume of DNPH reagent $(0.2 \% \mathrm{w} / \mathrm{v}$ in $2 \mathrm{~N} \mathrm{HCl})$ and allowed to stand for 30 minutes at $37^{\circ} \mathrm{C}$ or at least two hours at room temperature. The derivatives were extracted successively into isopropyl ether $(2 \times 5 \mathrm{ml}), 0.1 \mathrm{M} \mathrm{NaHCO}(3 \mathrm{ml})$, and finally, after acidification, into ethyl acetate $(6 \mathrm{ml})$ as described by Coward and Smith (1968). With normal urines aliquots of the final extracts equivalent to $2 \mathrm{mg}$ urinary creatinine were chromatographed but with the majority of the pathological urines described distortions due to overloading dictated the use of smaller samples, usually equivalent to 0.1 to $0.5 \mathrm{mg}$ urinary creatinine.

The samples were applied to $23 \times 23 \mathrm{~cm}$ sheets of Whatman no. SG81 paper and developed first with 
2-nitropropane containing $1 \%$ by volume of formic acid (solvent A) and then at right angles with benzene containing $10 \%$ by volume of acetic acid (solvent B). With this size paper chromatography occupied about six hours; useful preliminary information could be obtained in as little as one hour if smaller sheets of paper $(11 \times$ $11 \mathrm{~cm}$ ) were employed.

\section{RESULTS}

PHENYLKeTONURIA A typical case of phenylketonuria is illustrated in Figure 1. Three major spots, corresponding to the derivatives of phenylpyruvic, $p$-hydroxyphenylpyruvic, and $a$-ketoglutaric acids were observed, all being very much larger than spots observed in similar positions on chromatograms from normal urines. Each spot was eluted with $5 \%$ aqueous $\mathrm{NaHCO}_{3}$, extracted after acidification into ethyl acetate and finally reduced electrolytically (Smith and Smith, 1960) in acetic acid solution to give the corresponding aminoacid. Phenylalanine, tyrosine, and glutamic acid were identified by paper chromatography in n-butanol-acetic-acid water $(12: 3: 5)$ and by high-voltage electrophoresis in acetic acid-formic acid, $p \mathrm{H} 2$, and in pyridine acetate buffer, $p \mathrm{H} 4 \cdot 4$. The identity of the $p$-hydroxyphenylpyruvic acid derivative was further confirmed by its reaction with 2,6-dichloroquinonechloroimideborate to give a blue dyestuff.

Normal urines showed a weak spot in the position occupied by the DNP of phenylpyruvic acid; this

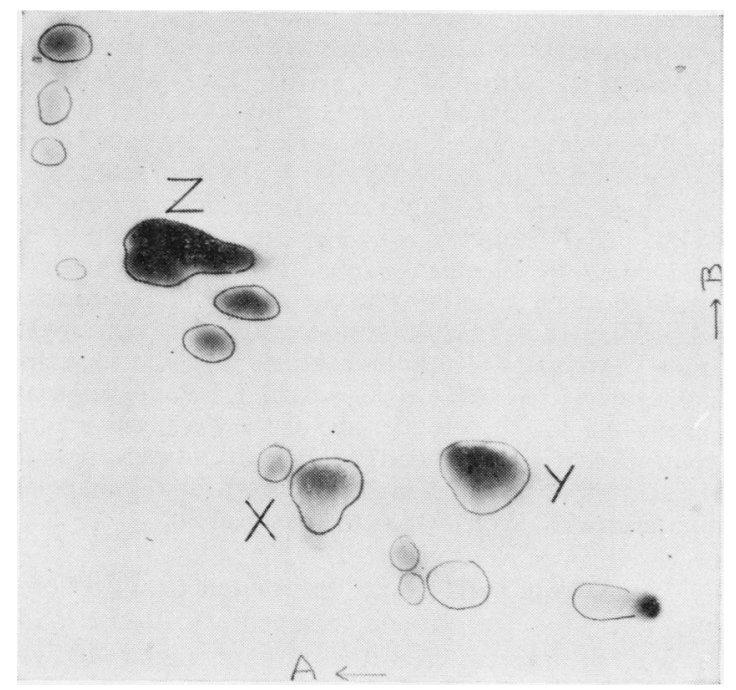

FIG. 1. 2,4-Dinitrophenylhydrazone chromatogram from a case of phenylketonuria. The extract chromatographed was equivalent to $0.1 \mathrm{mg}$ urinary creatinine.

$X$, p-hydroxyphenylpyruvic acid; $Y$, a-ketoglutaric acid; $Z$, phenylpyruvic acid (note double spot). spot is probably a mixture of the derivatives of higher aliphatic homologues of pyruvic acid which are not separable under the conditions of chromatography (Smith, 1967). In phenylketonuria phenylpyruvic acid sometimes gave a double spot (Fig. 1).

Urines from a total of 12 cases of phenylketonuria were examined. The excretion of phenylpyruvic acid varied greatly from subject to subject but was always grossly abnormal; in some cases urines gave a doubtful or negative response to the usual test with Phenistix and did not give an appreciable visible precipitate with the DNPH reagent.

In addition to phenylpyruvic acid a variety of other ketones often appeared to be excreted in large amounts in phenylketonuria. Most of these could not be correlated with known $\alpha$-ketoacids previously examined (Smith, 1967) and some were apparently not carboxylic acids since, after extraction with $\mathrm{NaHCO}_{3}$, further quantities could be extracted from the isopropyl ether solutions with methylamine. The derivative of $p$-hydroxyphenylpyruvic acid always showed as a strong, often major, spot. There was a tendency for excretion of $\alpha$-ketoglutaric acid to be high and in three subjects it was clearly grossly abnormal.

TYROSYLURIA The subject illustrated in Fig. 2 excreted, when 5 months old, large quantities of tyrosine and its metabolites, including $p$-hydroxyphenylpyruvic acid. At that time she had clinical symptoms which fulfilled the diagnostic criteria for tyrosinosis (Gjessing, 1966).

Numerous urine samples were studied, including a series collected after oral administration of L-phenylalanine $(0 \cdot 3 \mathrm{~g} / \mathrm{kg})$ to the subject. Figure 2 (I) shows a chromatogram from the sample collected four to eight hours after the administration of the aminoacid. Excretion of $p$-hydroxyphenylpyruvic acid was then somewhat greater than in control samples but the most interesting feature was the absence of any detectable amount of phenylpyruvic acid which would normally be expected to have been formed and excreted under the experimental conditions. Excretion of $\alpha$-ketoglutaric acid was high in urines from this subject. After she had been placed on a diet low in phenylalanine and tyrosine, excretion of $p$-hydroxyphenylpyruvic acid became normal and that of $a$-ketoglutaric acid was reduced to about 20 to $25 \%$ of previous levels as illustrated in Figure 2 (II).

After 10 months of dietary treatment urines from this subject remained normal in all respects when the intake of aromatic aminoacids was increased to normal levels. The phenylalanine loading test was then repeated but only in the four- to eight-hour sample was excretion of $p$-hydroxyphenylpyruvic 


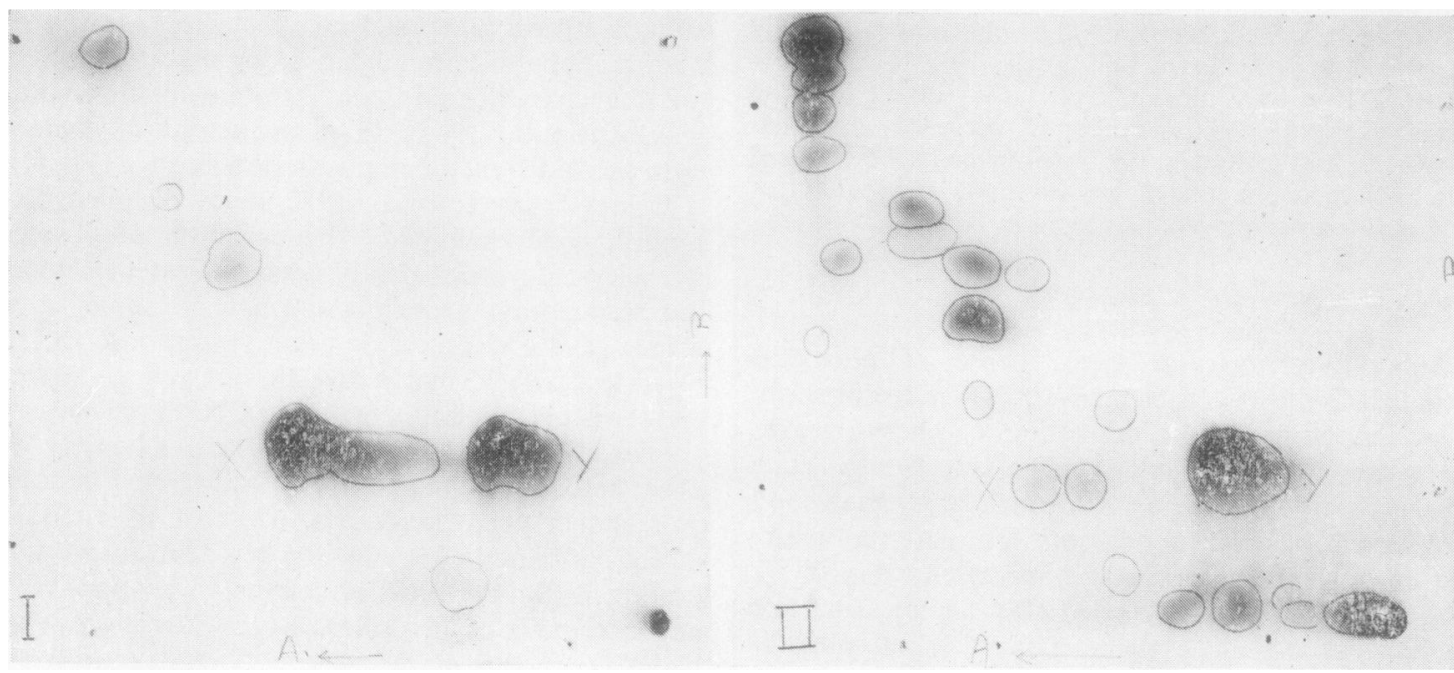

FIG. 2. 2,4-Dinitrophenylhydrazone chromatographs from a case of gross tyrosyluria, showing (I) an aliquot equivalent to $0.1 \mathrm{mg}$ urinary creatinine from a sample collected four to eight hours after administration of a phenylalanine load; (II), equivalent to $0.5 \mathrm{mg}$ creatinine, is from a sample collected while the subject was on a diet low in tyrosine and phenylalanine content.

acid increased to a level which, although greatly raised, was far less than that observed before treatment began. Further, in the same sample, a large spot due to phenylpyruvic acid was observed. The subject thus appeared to have suffered from a temporary coincidence of the clinical and biochemical symptoms characteristic of tyrosinosis (Harries, Seakins, Ersser, and Lloyd, 1969).

A further five infants with gross tyrosyluria, four of whom were ill, were examined. All excreted large amounts of $p$-hydroxyphenylpyruvic acid and, as in phenylketonuria, excretion of other ketones, mostly unidentified but including $\alpha$-ketoglutaric acid, was frequently high. One patient had a raised level of plasma methionine but we were not able to detect the corresponding $\alpha$-keto- $\gamma$-methylthiol-n-butyric acid in his urine. In two of the six cases excretion of p-hydroxyphenylpyruvic acid was not indicated by the Phenistix test and in one case the result was inconclusive.

HISTIDINAEMIA Urine from a case of histidinaemia, examined by the usual procedure, appeared to be quite normal except for the presence of one large DNP spot having approximate $R_{f}$ values of 0.45 and 0.05 in solvents A and B respectively. This compound gave a characteristic intense brown colour when exposed to methylamine vapour. A similar spot was often noticed in tyrosyluria, phenylketonuria, and other pathological urines.

The basic DNP of imidazolepyruvic acid is not extracted from acid solution into isopropyl ether or ethyl acetate and was not therefore present on the chromatograms from this subject. It is, however, extracted into $\mathrm{n}$-butanol and this property enabled its detection by the following procedure.

The urine-DNPH mixture $(2 \mathrm{ml})$ was shaken with n-butanol $(2 \mathrm{ml})$ and centrifuged. The upper layer was removed, evaporated to dryness in vacuo and the residue dissolved in formic acid $(200 \mu \mathrm{l})$. The derivative of imidazolepyruvic acid could be detected when aliquots were chromatographed on Whatman no. 20 paper using isopropanol-ammonia-water $(8: 1: 1)$ but was better observed after two-dimensional chromatography on SG81 paper. Aliquots equivalent to urine excreted in $0.05 \mathrm{~min}$ were chromatographed using solvent $\mathrm{A}$ which removed much material from the origin without moving imidazolepyruvic acid. Chromatography at right angles using nitromethane-formic acid $(9: 1)$ then revealed imidazolepyruvic acid as its yellow DNP, $\mathbf{R}_{\mathrm{f}}$ approximately 0.5 to 0.6 ; the identity of the spot was readily confirmed by spraying with alkaline diazotized sulphanilic acid with which it gave a red dyestuff, rapidly changing to a deep reddish-brown as the paper dried.

Urines from this subject responded to the Phenistix test only after he had received a histidine load. It was of interest that the patient (aged $10 \mathrm{yr}$ ) avoided meat, a rich source of histidine, of his own volition.

\section{DISCUSSION}

The basic technique proved to be of considerable 
value in studies of the effects of dietary changes as well as in the initial detection of ketoacidurias. The simplicity with which DNP extracts could be prepared and the rapidity of chromatography left little to be desired. In favourable cases, when urines gave a copious precipitate on the addition of DNPH reagent, this could be removed by centrifugation dissolved in a little ethyl acetate and immediately chromatographed. Preliminary small-scale paper chromatograms could thus be obtained within 90 minutes of the receipt of urine samples. The DNPH test (together with the Phenistix test) is not completely reliable and may fail with urines which nevertheless contain abnormal quantities of ketoacids. (A false positive may also result when subjects have received supplementary ascorbic acid.) If a ketoaciduria is suspected the urine should certainly be examined by the complete procedure; the greatly enhanced sensitivity conferred by chromatographic separation of compounds constitutes the fundamental advantage of the techniques. The method proved suitable for most ketoacidurias but required modification in the case of histidinaemia owing to the basic properties of imidazolepyruvic acid. No interference from acetoacetate was noted since the DNP of this acid decomposed to that of acetone under the experimental conditions.

The disadvantages of orthodox one-dimensional chromatography appeared largely to be overcome in the present method. It is true that an erratic tendency for some derivatives to give double spots was noticed, as in the cases of pyruvic acid (Coward and Smith, 1968) and phenylpyruvic acid (Fig. 1), but the phenomenon did not appear to be general and when two spots were given by one compound they were very close together if not actually merged. Ambiguities in identification of spots due to overlapping are much less serious in two- rather than one-dimensional procedures, but nevertheless still exist. Thus the one case of maple syrup urine disease that we have examined yielded a chromatogram similar to those obtained in phenylketonuria, since higher homologues of pyruvic acid yield derivatives having $R_{\mathbf{f}}$ values close to that of phenylpyruvic acid (Smith, 1967). Ambiguities of this type may be readily resolved, however, probably on clinical evidence and certainly by elution of the derivatives from paper followed by their reduction to readily identified aminoacids. Moreover in several cases pathologically interesting DNPs contain functional groups which respond to specific reagents which may be used for confirmatory tests. Such compounds include the derivatives of $p$-hydroxyphenylpyruvic acid and 4-hydroxy-3-methoxyphenylpyruvic acid which react with diazotized amines and 2 :6-dichloroquinonechloroimide, imidazole- pyruvic acid (diazotized amines), indolepyruvic acid (Ehrlich's reagent), and $a$-keto- $\gamma$-methylthiol-nbutyric acid (iodoplatinate reagent).

One of the major advantages of chromatographic techniques is that they invariably yield information concerning substances other than those of immediate interest to the investigator. The present technique proved no exception to this rule and many pathological urines were observed to contain large amounts of unexpected compounds, some of which were identified with known $\alpha$-ketoacids. When, as in phenylketonuria and tyrosyluria, large quantities of an $\alpha$-ketoacid accumulate in the blood, it might be expected to undergo, on mass-action principles, transamination with $\alpha$-aminoacids leading to increased production and excretion of their corresponding $\alpha$-ketoacids. Such may well account for our observation of high excretion of $\alpha$-ketoglutaric acid in these conditions, since this acid with its corresponding $\alpha$-aminoacid (glutamic acid) plays a key role in transamination processes. The well known excretion of $p$-hydroxyphenyllactic and indolelactic acids in phenylketonuria has previously led to the suspicion that the corresponding pyruvic acids should also be excreted in increased amounts. However, Armstrong (1963) was unable to confirm reports that $p$-hydroxyphenylpyruvic acid (Rees, 1955) and indolepyruvic acid (Schreier and Flaig, 1956) are indeed excreted. We have now been able to show beyond doubt that $p$-hydroxyphenylpyruvic acid is present in phenylketonuric urine though we were not able to demonstrate the presence of indolepyruvic acid. In normal subjects $p$-hydroxyphenylpyruvic acid is oxidized almost completely to homogentisic acid. When, as in tyrosyluria, the enzyme $p$-hydroxyphenylpyruvic acid oxidase which is responsible for this oxidation is deficient in activity, $p$-hydroxyphenylpyruvic acid accumulates and is excreted in abnormal quantities, together with a reduction product, $p$-hydroxyphenyllactic acid. Zannoni and La Du (1959) found the enzyme to be inhibited by phenylpyruvic acid and this effect rather than, or in addition to, a transamination reaction, may account for the excretion of $p$-hydroxyphenylpyruvic acid in phenylketonuria.

Treatment of both phenylketonuria and tyrosinosis, a potentially fatal form of tyrosyluria (Gjessing, 1966), depends upon the administration of diets containing minimal amounts of the relevant aminoacids. The implicit suggestion that toxic metabolites are therefore involved in both diseases has not been supported by the positive identification of any compound possessing the necessary toxicity. Our observation that unidentified ketones are often excreted in ketoacidurias is therefore of interest in that it emphasizes that there are biochemical 
features of these diseases which have not as yet been investigated.

\section{REFERENCES}

Armstrong, M. D. (1963). In Phenylketonuria, edited by F. L. Lyman, p. 92. C. C. Thomas, Springfield, Illinois.

Coward, R. F., and Smith, P. (1968). J. Chromatog., 33, 508.

Gjessing, L. R., ed. (1966). Symposium on Tyrosinosis, 1965. Universitatsforlaget, Oslo.
Harries, J. T., Seakins, J. W. T., Ersser, R. S., and Lloyd, J. K. (1969). Arch. Dis. Childh., 44, 258.

Rees, K. R. (1955). Cited by Bickel, H., Boscott, R. J. and Gerrard.

J. In Biochemistry of the Developing Nervous System, edited by H. Waelsch, p. 417. Academic Press, New York.

Schreier, K., and Flaig, H. (1956). Klin. Wchnschr., 34, 1213.

Smith, I., and Smith, M. (1960). In Chromatographic and Electrophoretic Techniques, edited by I. Smith, vol. I, p. 261. Heinemann, London.

Smith, P. (1967). J. Chromatog., 30, 273.

Zannoni, V. G., and La Du, B. N. (1959). J. biol. Chem., 234, 2925. 